\title{
Bipolar Disorder Comorbidity in Anxiety Disorders: Relationship to demographic profile, symptom severity, and functional impairment
}

\author{
Katie A. Fracalanza, MA* \\ Randi E. McCabe, PhD $D^{\star *, * * *}$

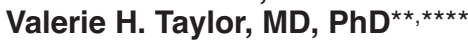 \\ Martin M. Antony, $\mathrm{PhD}^{*, * * *}$ \\ * Department of Psychology, Ryerson \\ University, Toronto, Ontario \\ ** Department of Psychiatry and \\ Behavioural Neurosciences, McMaster \\ University, Hamilton, Ontario \\ *** Anxiety Treatment and Research \\ Centre, St. Joseph's Healthcare Hamilton, \\ Hamilton, Ontario

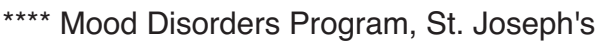 \\ Healthcare Hamilton, Hamilton, Ontario
}

CANADA

\begin{abstract}
Background and Objectives: High rates of comorbidity between anxiety disorders and bipolar disorder (BD) have been reported. Studies on the impact of BD comorbidity in individuals with a principal anxiety disorder have been limited.

Methods: Individuals $(N=186)$ seeking anxiety disorder treatment completed questionnaires and a diagnostic interview. Anxious individuals with comorbid BD were compared to anxious individuals with comorbid depression, and individuals with an anxiety disorder only.

Results: Anxious individuals with BD were more likely to report being single, separated or divorced, and to present with greater substance abuse and comorbidity than individuals with an anxiety disorder only. Anxious individuals with BD also presented with lower household income than anxious individuals with depression, and individuals with anxiety only. Anxious individuals with either comorbid BD or comorbid depression reported greater functional impairment and more severe symptoms than individuals with anxiety only.

Conclusions: Overall, the presence of comorbid BD was associated with demographic and clinical factors that have been previously shown to adversely affect treatment outcome in people with anxiety disorders.
\end{abstract}




\section{Introduction}

Relatively few studies have investigated the relationship between anxiety disorders and bipolar disorder, particularly when anxiety is the principal diagnosis. This limitation in the literature is problematic, as anxiety disorders and bipolar disorder commonly co-occur. In bipolar disorder samples, lifetime frequency estimates for the occurrence of any anxiety disorder range from $24 \%{ }^{1}$ to $79 \%^{2}$, whereas the lifetime prevalence of anxiety disorders in the general population was estimated to be $29 \%$ in the most recent National Comorbidity Survey ${ }^{3}$. Likewise, in anxiety disorders samples, the lifetime frequency of bipolar disorder ranges from $6 \% 4$ to $26 \%{ }^{5}$, which is higher than the lifetime prevalence of $3.9 \%$ in the community ${ }^{3}$.

The high frequency of anxiety disorders among individuals with bipolar disorder is concerning, as comorbid anxiety disorders have been linked to numerous complications in this group. These problems include a greater risk of substance use disorders ${ }^{6}$, eating disorders ${ }^{7}$, and suicidal behavior ${ }^{8}$. Additionally, bipolar disorder patients with a comorbid anxiety disorder have an earlier age of bipolar disorder onset ${ }^{9,10}$, more severe depressive symptoms ${ }^{11}$, greater functional impairment ${ }^{6,12}$, lower quality of life ${ }^{13}$, and poorer treatment outcomes ${ }^{6,14}$. Overall, comorbid anxiety disorders have a negative impact on the course and treatment response in bipolar disorder.

Further, comorbid bipolar disorder has been associated with complications in individuals with anxiety disorders. One study found that social anxiety disorder patients with comorbid bipolar disorder presented with more severe social anxiety symptoms, a greater degree of comorbidity, and greater alcohol abuse than social anxiety patients without comorbid bipolar disorder ${ }^{15}$. Anoth- er study found that obsessive-compulsive disorder (OCD) patients with comorbid bipolar disorder have higher lifetime rates of substance use disorders ${ }^{16}$ than OCD patients without comorbid bipolar disorder. Even comorbid cyclothymic disorder, which is characterized by less severe hypomanic and depressive symptoms than bipolar disorder, has been associated with a range of complications in OCD patients ${ }^{17}$. The purpose of the current study was to examine the impact of comorbid bipolar disorder across the anxiety disorders. The specific aims of this study were to determine: (1) which principal anxiety disorders individuals with comorbid bipolar disorder seek treatment for; (2) whether individuals with an anxiety disorder and comorbid bipolar disorder present with unique sociodemographic and clinical characteristics; and (3) whether individuals with an anxiety disorder and comorbid bipolar disorder present with a greater degree of symptom severity and functional impairment than individuals without bipolar disorder. To ensure that our findings were specific to bipolar disorder and not simply to the presence of depression we included a comparison group of individuals with an anxiety disorder and comorbid unipolar depression.

\section{Method}

\section{Participants}

The sample consisted of 186 individuals referred for treatment at the Anxiety Treatment and Research Centre (ATRC), a specialized outpatient anxiety disorders clinic in Hamilton, Ontario, Canada. This study compared three groups: (1) individuals with a principal anxiety disorder and comorbid bipolar disorder (ANX + BD; $n=62)$, (2) individuals with a principal anxiety disorder 
and comorbid unipolar depression (ANX + $\mathrm{D} ; n=62$ ), and (3) individuals with a principal anxiety disorder and no mood disorder (ANX; $n=62$ ).

The three groups were selected from a database of 1019 individuals referred for treatment at the ATRC between 2002 and 2007. Individuals were excluded from the ANX + BD group if they had bipolar disorder in partial or full remission, as defined by the criteria in the Diagnostic and Statistical Manual of Mental Disorders (DSM-IV-TR) ${ }^{18}$, at the time of assessment. Restricting the sample to individuals with current diagnoses was done to compare the impact of active symptoms between groups. Within the ANX + BD group, 71\% had bipolar I disorder, $27 \%$ had bipolar II disorder, and $2 \%$ had bipolar disorder not otherwise specified. Of those with bipolar I disorder, 91\% were experiencing a depression phase of bipolar illness at the time of assessment, 7\% were experiencing a mixed episode, and $2 \%$ had experienced a manic or hypomanic episode in the two months prior to assessment. Individuals in the comorbid unipolar depression $(\mathrm{ANX}+\mathrm{D})$ group had a current diagnosis of major depressive disorder, as defined by DSM-IV-TR ${ }^{18}$.

Individuals in the two comparison groups (ANX + D and ANX) were selected to match the ANX + BD group based on principal anxiety disorder, sex, and age. All comparison individuals had the same principal anxiety disorder as the bipolar disorder individual that they were matched to. Comparison individuals were age and sex matched as closely as possible to bipolar disorder individuals. Most (96\%) of the comparison individuals were of the same sex as the bipolar disorder individual that they were matched to, and the majority (89\%) were age-matched within two years. There was a maximum difference of eight years between bipolar disor- der individuals and comparison individuals. When two or more comparison individuals were deemed to be an appropriate match for a bipolar disorder patient, the comparison case with the lowest patient identification number was selected to ensure that the comparison groups were chosen without bias.

\section{Measures}

Structured Clinical Interview for DSM-IV (SCID-IV) ${ }^{19}$. The SCID-IV is a semi-structured interview designed to assess for the most common Axis I disorders according to DSM-IV criteria ${ }^{18}$. Earlier versions of the SCID have demonstrated good reliability for all disorders across a variety of samples ${ }^{20,21}$ and adequate test-retest reliability ${ }^{21,22}$.

Depression Anxiety Stress Scales 21- Item Version (DASS-21) ${ }^{23}$. The DASS-21 is comprised of three subscales. The depression subscale (DASS-D) assesses dysphoric mood; the anxiety subscale (DASS-A) measures fear symptoms and autonomic arousal; and the stress subscale (DASS-S) is sensitive to non-specific symptoms of tension and agitation. The DASS-21 has high internal consistency, and high convergent and discriminant validity in both community ${ }^{24}$ and clinical samples ${ }^{23,25}$.

Illness Intrusiveness Ratings Scale (IIRS) ${ }^{26}$. The IIRS measures the impact of an illness and its treatment across various life domains, such as work and relationships. This questionnaire has high internal consistency and high inter-item correlations ${ }^{27}$.

\section{Procedure}

Use of the data from the ATRC research database was approved by the Research Ethics Board at St. Joseph's Healthcare Hamilton. Individuals were referred to the ATRC by a 
physician. To determine diagnoses, each person was interviewed by a trained clinician using the SCID-IV. All diagnoses were reviewed by a psychologist with more than five years of experience in administering the SCID-IV. When multiple disorders were present, the disorder causing the greatest amount of distress or impairment was considered the principal diagnosis. Individuals completed the DASS-21 and IIRS as part of a general questionnaire intake package.

\section{Overview of Statistical Analyses}

Statistical analyses were performed using the Statistical Package for Social Sciences (SPSS) version 17.0 (SPSS, Chicago, IL). The data analytic strategies paralleled the specific goals of the study. Between-group (three levels: $\mathrm{ANX}+\mathrm{BD}, \mathrm{ANX}+\mathrm{D}, \mathrm{ANX})$ differences in sociodemographic and clinical characteristics were assessed using a series of one-way Analyses of Variance (ANOVAs) for continuous variables, and a series of chi-square tests for categorical variables. Significant omnibus results were followed by Bonferroni post-hoc tests for continuous variables, and pairwise Mann-Whitney U tests for categorical variables. Between-group differences in symptom severity and functional impairment were assessed using a series of Analyses of Covariance (ANCOVAs), controlling for the number of diagnoses in addition to mood disorders. Significant ANCOVAs were followed by Bonferroni post-hoc tests. All $p$ values were 2-tailed, and statistical significance was set at $p<0.05$.

\section{Results}

\section{Presenting Anxiety Disorders in Individuals with Comorbid Bipolar Disorder}

In the current sample of individuals with comorbid bipolar disorder, social anxiety disorder was the most frequently observed anxiety disorder (39\%), followed by panic disorder (31\%), and obsessive-compulsive disorder $(16 \%)$. More information can be found in Table 1. Of the group of individuals with a principal anxiety disorder and comorbid bipolar disorder, $30 \%$ had one additional anxiety disorder diagnosis, $19 \%$ had two additional anxiety disorder diagnoses, and $21 \%$ had three or more additional anxiety disorder diagnoses.

Table 1

Current Principal Anxiety Disorder Diagnoses for 62 individuals with Comorbid Bipolar Disorder

Principal Anxiety Disorder Diagnosis

Bipolar Disorder Group $(n=62)$

\begin{tabular}{lcc}
\hline & $\mathrm{n}$ & $\%$ \\
\hline Social Anxiety Disorder & 24 & 39 \\
Panic Disorder & 19 & 31 \\
Obsessive-Compulsive Disorder & 10 & 16 \\
Generalized Anxiety Disorder & 6 & 10 \\
Posttraumatic Stress Disorder & 2 & 3 \\
Anxiety Disorder Not Otherwise Specified & 1 & 1 \\
\hline
\end{tabular}

Note. Panic Disorder includes individuals with and without agoraphobia. 


\section{Sociodemographic and Clinical Characteristics of Individuals with Comorbid Bipolar Disorder}

The sociodemographic characteristics of the sample are presented in Table 2. The ANX+BD group was significantly more likely to report being single, separated, or divorced than the ANX group, $\mathrm{U}=1435, z=$ $-2.52, p=0.02$. The ANX+D group did not differ from either group on marital status. The ANX+BD group reported significantly lower household income than the ANX group, $\mathrm{U}=1113, z=-3.49, p=0.01$, and the ANX+D group, $\mathrm{U}=1272, z=-2.05, p=0.05$. In terms of clinical characteristics, there was a significant difference in the number of comorbid diagnoses between groups, $F$ $(2,183)=4.27, p=0.02$. Bonferroni post hoc tests indicated that the ANX+BD group reported a significantly higher number of additional comorbid diagnoses than did the ANX group, while ANX+D group did not differ from either group. To determine if there were group differences in comorbid alcohol and substance use disorders, individuals' responses to the SCID-IV were examined. The ANX+BD group was significantly more likely to report an alcohol or substance use disorder at initial presentation than the ANX group, $\mathrm{U}=1612, z=-3.03, p=0.01$. The ANX+D group did not differ from either group on alcohol or substance use.

\section{Symptom Severity and Functional Impairment}

Controlling for the number of diagnoses in addition to a mood disorder, the ANX + $\mathrm{BD}$ and $\mathrm{ANX}+\mathrm{D}$ groups reported higher scores than the ANX group on the depression subscale of the DASS- $21, F(2,181)=$ $18.79, p=0.01$, and the anxiety subscale of the DASS-21, $F(2,181)=7.95, p=0.01$.
There were no significant differences between groups on the stress subscale of the DASS-21. The ANX + BD and ANX + D groups reported higher scores than the ANX group on the IIRS, $F(2,182)=8.20, p=$ 0.01 . The means and standard deviations of scores on the DASS-21 and IIRS are presented in Table 3.

\section{Discussion}

The present study sought to provide information about the demographic and clinical profile of individuals presenting with a principal anxiety disorder and comorbid bipolar disorder. Our findings showed that social anxiety disorder was the most common presenting anxiety disorder in individuals with comorbid bipolar disorder. In terms of demographic characteristics, individuals with comorbid bipolar disorder were more likely to report being single, separated or divorced than individuals with an anxiety disorder only. Further, individuals with comorbid bipolar disorder were more likely to report a lower household income than individuals with comorbid unipolar depression or an anxiety disorder only. Individuals with comorbid bipolar disorder also present with unique clinical characteristics. Compared to individuals with an anxiety disorder alone, individuals with bipolar disorder were significantly more likely to report: (1) a greater number of additional diagnoses (not including bipolar disorder), and (2) a comorbid substance use disorder. Controlling for the number of comorbid diagnoses, individuals with comorbid bipolar disorder or comorbid unipolar depression presented with more severe depression and anxiety symptoms, and reported greater functional impairment than individuals with an anxiety disorder alone. 


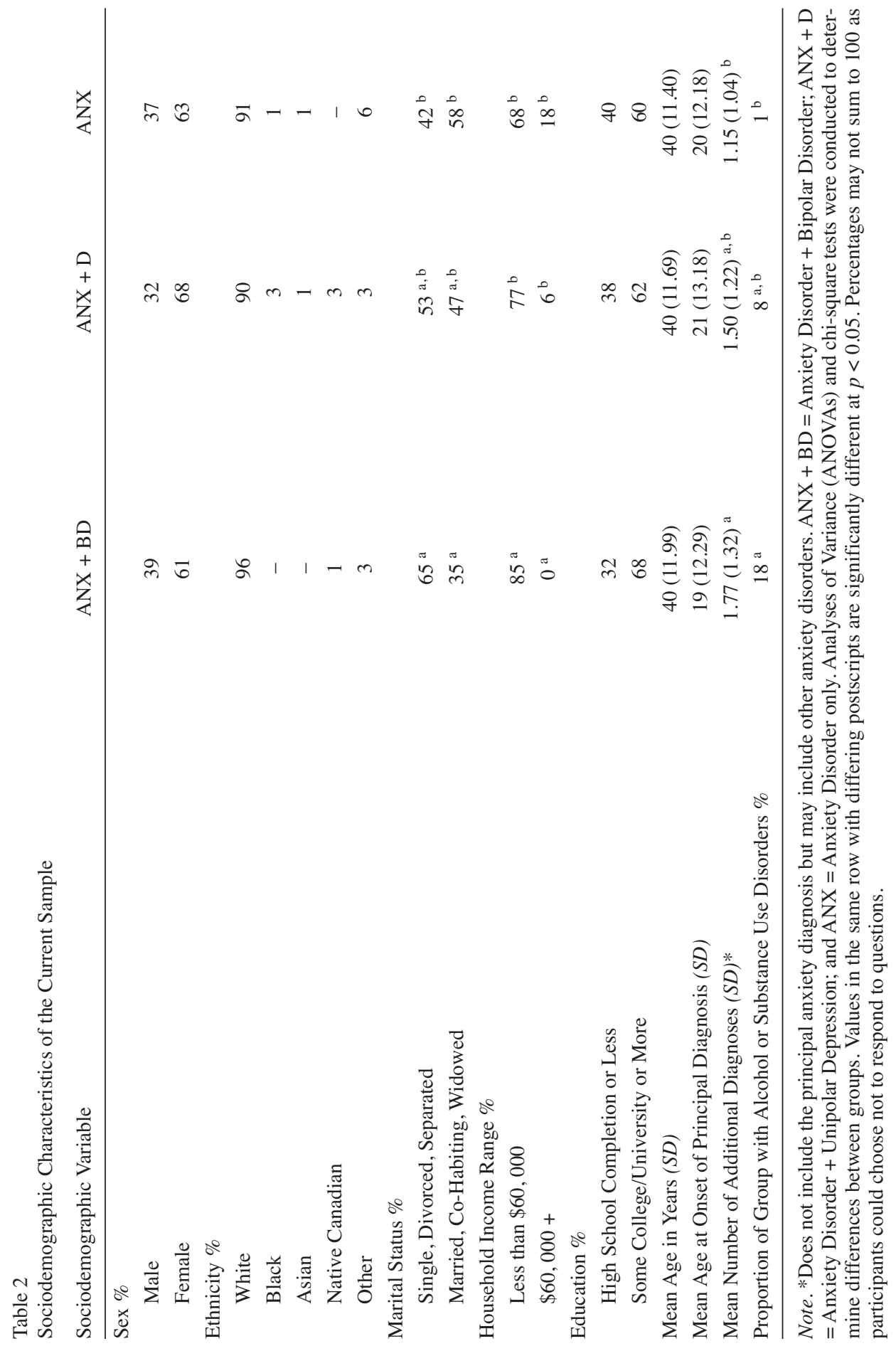




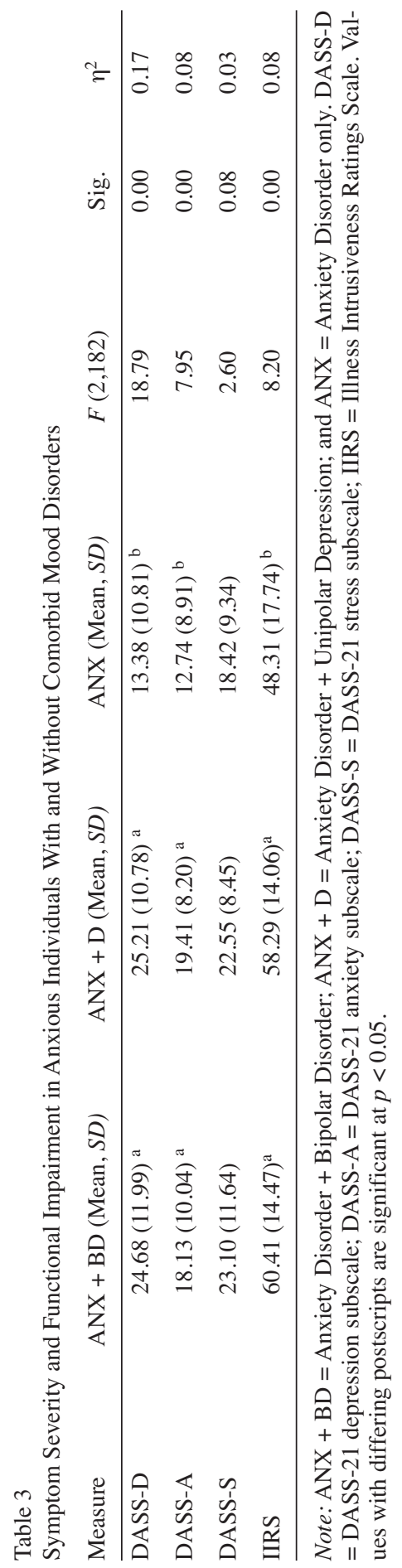

The finding that anxious individuals with comorbid bipolar disorder were more likely to report being single, separated, or divorced is consistent with findings from prior epidemiological studies on marital status and bipolar disorder. The Epidemiologic Catchment Area (ECA) study found that bipolar disorder was reported more frequently by individuals who were divorced or never married compared to individuals who were married $^{28}$. In line with this, the National Comorbidity Survey found that being divorced or separated was a sociodemographic risk factor associated with a greater likelihood of any mood or anxiety disorder ${ }^{3}$. Additionally, a large epidemiological study which examined the relationship between marital distress and DSM-IV psychiatric disorders found that bipolar disorder was more strongly associated with marital distress than was any other psychiatric disorder ${ }^{29}$. It is possible greater marital distress accounts for the lower likelihood of being married reported by individuals with comorbid bipolar disorder in the present study. These findings are notable, as being married is a protective demographic factor that has been linked to better treatment outcome ${ }^{30}$.

In contrast to the current findings, comorbid bipolar disorder has not been previously linked to marital status in other anxiety disorders samples. No differences in marital status were found in social anxiety disorder patients with and without bipolar disorder ${ }^{15}$. Similarly, previous research found no differences in marital status in OCD patients with remitted bipolar disorder versus those with OCD only ${ }^{31}$. Methodological and sample differences between the current study and past research may account for these inconsistent findings (for example, individuals with remitted bipolar disorder were excluded from the current study). Despite the specific association between marital status 
and bipolar disorder found in the current study, it is possible that bipolar disorder as a comorbid condition does not have the same detrimental impact on marital status as bipolar disorder assigned as a principal diagnosis. Further research on comorbid bipolar disorder and marital status is necessary to clarify these inconsistent results.

The difference in income level between individuals with comorbid bipolar disorder and both comparison groups is in line with prior research showing that bipolar disorder is linked to low income. One study examining the demographic characteristics of individuals with bipolar disorder found that more than half of the sample had low household income $(<\$ 20,000$ per year), despite high education levels ${ }^{32}$. One reason for low income in individuals with bipolar disorder is lower rates of full-time employment ${ }^{33-35}$. The chronic and episodic course of bipolar disorder makes it more likely that individuals affected by this disorder will have gaps in work attendance and quality of work performance. Overall, the income demographics found in the current study are in line with the limitations in functioning that have been consistently associated with bipolar disorder in prior research ${ }^{35}$.

In the present study, individuals with comorbid bipolar disorder presented with a greater number of additional disorders (over and above bipolar disorder) than individuals with an anxiety disorder alone. In addition, comorbid alcohol and substance use disorders were significantly more frequent in individuals with bipolar disorder compared to individuals with an anxiety disorder only. Past studies of non-anxious bipolar disorder patients report that alcohol and substance abuse are the most common psychological comorbidities ${ }^{36}$. Additionally, bipolar disorder - anxiety disorder comorbidity has been associated with substance use problems in previous research in bipolar disorder samples $^{6}$ and anxiety disorder samples ${ }^{37}$. It seems that individuals with bipolar - anxiety disorder comorbidity are at higher risk for substance abuse regardless of their principal diagnosis. Further, a specific link between social anxiety disorder, alcohol abuse, and bipolar II disorder has been proposed in past research ${ }^{37}$. The common occurrence of social anxiety disorder and substance abuse in individuals with bipolar disorder in the current sample is consistent with this hypothesis. The high frequency of substance abuse in anxious bipolar disorder individuals may negatively impact anxiety treatment. Intoxication during therapy sessions can interfere with the activation of anxiety, which is thought to be necessary for many evidence-based treatments for anxiety reduction (e.g., cognitive behavioral therapy $)^{38}$. These findings underline the importance of assessing for comorbid substance abuse in anxious individuals prior to treatment, and addressing this at the outset of treatment if necessary.

After controlling for comorbidity, both individuals with either comorbid bipolar disorder or comorbid depression had more severe depression and anxiety symptoms, as well as greater functional impairment compared to individuals with an anxiety disorder alone. The bipolar and depression groups may not have differed on these variables as most individuals in the present bipolar disorder sample were in the depressed phase of bipolar illness. Our findings suggest that when symptom severity and functional impairment are measured during the depression phase of bipolar disorder, anxious bipolar disorder individuals are not more impaired than anxious depressed individuals. However, anxious individuals with either mood disorder were more impaired than anxious individuals without a mood disorder. The 
finding that individuals with bipolar disorder have more severe depression and anxiety symptoms is noteworthy, as individuals with more severe global symptoms are poorer responders to group cognitive behavioural therapy for anxiety ${ }^{39}$. Clinicians should be mindful of these symptoms and provide adjunct treatment aimed at relieving severe depressive symptoms in addition to group treatment for anxiety if warranted.

The present findings must be interpreted in light of several limitations. First, the questionnaires used to determine symptom severity and functional impairment were self-report measures. Confidence in these findings would be strengthened by adding clinician-administered measures and objective measures of impairment. Additionally, the frequency pattern of anxiety disorders found in the current sample may have been influenced by biases inherent in referrals to the ATRC. For example, the ATRC does not routinely offer treatment for PTSD, so it is likely that individuals with bipolar disorder and PTSD would be referred elsewhere. Finally, the generalizability of these results to the larger population of individuals with comorbid bipolar and anxiety disorders may be limited because the study was conducted at a specialized anxiety disorders clinic. This implies that the comorbid bipolar disorder was not the main treatment target of patients or their physicians. Thus the severity and complications associated with comorbid bipolar disorder in the present study may be an underestimate of that which may be associated with comorbid bipolar disorder in more general psychiatric settings. Generalizability is also limited by the fact that only individuals with symptoms meeting diagnostic criteria for current bipolar disorder were included in the study. Thus, the findings may not apply to individuals who present for anxiety treatment with bipolar disorder in partial or full remission.
This study examined the impact of bipolar disorder in an anxiety disorder sample prior to treatment. These findings suggest that before beginning treatment, individuals with bipolar disorder comorbidity present with several sociodemographic risk factors. Further, this group of individuals has a greater number of additional diagnoses, substance use disorders, and more severe symptoms and impairment than individuals presenting with anxiety disorders alone. This study serves as the basis for predicting that anxious bipolar disorder individuals may not fare as well in treatment as anxious-depressed individuals or individuals with anxiety only. Future research should investigate this hypothesis, in order to determine whether additional interventions would enhance the current empirically-supported protocols for treating anxiety disorders within the context of comorbid bipolar disorder.

\section{References}

1. Henry C, Van den Bulke D, Bellivier F, Etain B, Rouillon F, Leboyer M. Anxiety disorders in 318 bipolar individuals: Prevalence and impact on illness severity and response to mood stabilizer. J Clin Psychiatry 2003; 64(3): 331-335.

2. Pini S, Cassano GB, Simonini E, Savino M. Prevalence of anxiety disorders comorbidity in bipolar depression, unipolar depression and dysthymia. J Affect Disord 1997; 42(2-3): 145-153.

3. Kessler RC, Berglund P, Demler O, Jin R, Merikangas KR, Walters EE. Lifetime prevalence and age-of-onset distributions of DSM-IV disorders in the national comorbidity survey replication. Arch Gen Psychiatry 2005; 62(6): 593-602.

4. Perugi G, Akiskal HS, Ramacciotti S, Nassini S, Toni C, Milanfranchi A, et al. Depressive comorbidity of panic, social phobic, and obsessive-compulsive disorders re-examined: Is there a bipolar II connection? J Psychiatr Res 1999; 33(1): 53-61.

5. Goldstein BI, Levitt AJ. The specific burden of comorbid anxiety disorders and of substance use disorders in bipolar I disorder. Bipolar Disord 2008; 10(1): 67-78. 
6. Simon NM, Otto MW, Wisniewski SR, Fossey M, Sagduyu K, Frank E, et al. Anxiety disorder comorbidity in bipolar disorder individuals: Data from the first 500 participants in the systematic treatment enhancement program for bipolar disorder (STEP-BD). Am J Psychiatry 2004; 161(12): 2222-2229.

7. MacQueen GM, Marriott M, Begin H, Robb J, Joffe RT, Young LT. Subsyndromal symptoms assessed in longitudinal, prospective follow-up of a cohort of individuals with bipolar disorder. Bipolar Disord 2003; 5(5): 349-355.

8. Lee JH, Dunner DL. The effect of anxiety disorder comorbidity on treatment resistant bipolar disorders. Depress Anxiety 2008; 25(2): 91-97.

9. Bauer MS, Altshuler L, Evans DR, Beresford T, Williford WO, Hauger R, et al. Prevalence and distinct correlates of anxiety, substance, and combined comorbidity in a multi-site public sector sample with bipolar disorder. J Affect Disord 2005; 85(3): 301-315.

10. McElroy SL, Altshuler LL, Suppes T, Keck PE Jr, Frye MA, Denicoff KD, et al. Axis I psychiatric comorbidity and its relationship to historical illness variables in 288 individuals with bipolar disorder. Am J Psychiatry 2001; 158(3): 420-426.

11. Boylan KR, Bieling PJ, Marriott M, Begin H, Young LT, MacQueen GM. Impact of comorbid anxiety disorders on outcome in a cohort of individuals with bipolar disorder. J Clin Psychiatry 2004; 65(8): 1106-1113.

12. Keller MB, Krystal JH, Hen R, Neumeister A, Simon NM. Untangling depression and anxiety: Clinical challenges. J Clin Psychiatry 2005; 66(11): 1477-1484.

13. Albert U, Rosso G, Maina G, Bogetto F. Impact of anxiety disorder comorbidity on quality of life in euthymic bipolar disorder individuals: Differences between bipolar I and II subtypes. J Affect Disord 2008; 105(1-3): 297-303.

14. Tohen M, Calabrese J, Vieta E, Bowden C, Gonzalez-Pinto A, Lin D, et al. Effect of comorbid anxiety on treatment response in bipolar depression. J Affect Disord 2007; 104(1-3): 137-146.

15. Perugi G, Frare F, Toni C, Mata B, Akiskal HS. Bipolar II and unipolar comorbidity in 153 outindividuals with social phobia. Compr Psychiatry 2001; 42(5): 375-381.

16. Maina G, Albert U, Pessina E, Bogetto F. Bipolar obsessive-compulsive disorder and personality disorders. Bipolar Disord 2007; 9(7): 722-729.

17. D'Ambrosio V, Albert U, Bogetto F, Maina G. Obsessive-compulsive disorder and cyclothymic temperament: An exploration of clinical features. J Affect Disord 2010; 127(1-3): 295-299.
18. American Psychiatric Association. Diagnostic and statistical manual of mental disorders. 4 th ed., text revision. Washington; 2000.19. First MB, Spitzer RL, Gibbon M, Williams JBW. Structured Clinical Interview for DSMIV Axis I Disorders - Patient edition. SCID-I/P, Version 2.0. New York: Psychiatric Institute, Biometrics Research Department; 1996.

20. Segal DL, Hersen M, Van Hasselt VB. Reliability of the structured clinical interview for DSM-III-R: An evaluative review. Compr Psychiatry 1994; 35(4): 316-327.

21. Williams JB, Gibbon M, First MB, Spitzer RL. The structured clinical interview for DSM-III-R (SCID): II. multisite test-retest reliability. Arch Gen Psychiatry 1992; 49(8): 630-636.

22. Zanarini MC, Frankenburg FR. Attainment and maintenance of reliability of axis I and II disorders over the course of a longitudinal study. Compr Psychiatry 2001; 42(5): 369-374.

23. Lovibond SH, Lovibond PF. Manual for the Depression Anxiety Stress Scales. 2nd ed. Sydney: Psychology Foundation of Australia; 1995.

24. Crawford JR, Henry JD. The depression anxiety stress scales (DASS): Normative data and latent structure in a large non-clinical sample. Br J Clin Psych 2003; 42(2): 111-131.

25. Antony MM, Bieling PJ, Cox BJ, Enns MW, Swinson RP. Psychometric properties of the 42-item and 21item versions of the depression anxiety stress scales in clinical groups and a community sample. Psychol Assess 1998; 10(2): 176-181.

26. Devins GM, Binik YM, Hutchinson TA, Hollomby DJ, Barré PE, Guttmann RD. The emotional impact of endstage renal disease: Importance of individuals' perceptions of intrusiveness and control. Int J Psychiatr Med 1983; 13(4): 327-343.

27. Devins GM, Dion R, Pelletier LG, Shapiro CM, Abbey S, Raiz LR, et al. Structure of lifestyle disruptions in chronic disease: A confirmatory factor analysis of the illness intrusiveness ratings scale. Med Care 2001; 39(10): 1097-1104.

28. Weissman MM, Kidd KK, Prusoff BA. Variability in rates of affective disorders in relatives of depressed and normal probands. Arch Gen Psychiatry 1982; 39(12): 1397-1403.

29. Whisman MA. Marital distress and DSM-IV psychiatric disorders in a population-based national survey. J Abnorm Psychol 2007; 116(3): 638-643.

30. Meyers BS, Sirey JA, Bruce M, Hamilton M, Raue $\mathrm{P}$, Friedman SJ, et al. Predictors of early recovery from 
major depression among persons admitted to communitybased clinics: An observational study. Arch Gen Psychiatry 2002; 59(8): 729-735.

31. Zutshi A, Kamath P, Reddy YC. Bipolar and nonbipolar obsessive-compulsive disorder: A clinical exploration. Compr Psychiatry 2007; 48(3): 245-251.

32. Suppes T, Leverich GS, Keck PE, Nolen WA, Denicoff KD, Altshuler LL, et al. The Stanley Foundation Bipolar Treatment Outcome Network: II. Demographics and illness characteristics of the first 261 individuals. J Affect Disord 2001; 67(1-3): 45-59.

33. Kusznir A, Scott E, Cooke R, Young L. Functional consequences of bipolar affective disorder: An occupational therapy perspective. Can J Occup Ther 1996; 63(1): 313-322.

34. McPherson H, Dore G, Loan P, Romans S. Socioeconomic characteristics of a Dunedin sample of bipolar individuals. N Z Med J 1992; 105(993): 161-162.

35. Tsai S, Chen C, Yeh E. Alcohol problems and longterm psychosocial outcome in chinese individuals with bipolar disorder. J Affect Disord 1997; 46(2): 143-150.
36. Weissman MM, Johnson J. Drug use and abuse in five US communities. New York State J Med 1991; 91(11): 19-23.

37. Perugi G, Frare F, Madaro D, Maremmani I, Akiskal HS. Alcohol abuse in social phobic individuals: Is there a bipolar connection? J Affect Disord 2002; 68(1): 33-39.

38. Ramirez Basco M, Rush AJ. Cognitive-behavioral therapy for bipolar disorders. 2nd ed. New York: Guilford Publications; 2005.

39. Raffin AL, Fachel JMG, Ferrão YA, de Souza FP, Cordioli AV. Predictors of response to group cognitive-behavioral therapy in the treatment of obsessive-compulsive disorder. Eur Psychiat 2009; 24(5): 297-306.

Author for correspondence:

Dr. Randi E. McCabe

Anxiety Treatment and Research Centre

St. Joseph's Healthcare, 50 Charlton Avenue East

Hamilton, Ontario, Canada L8N 4A6

Tel: 905-522-1155, ext. 33695

Fax: 905-521-6120

E-mail: rmccabe@stjoes.ca 